\title{
MULTICULTURALISMO, relativismo y racionalidad
}

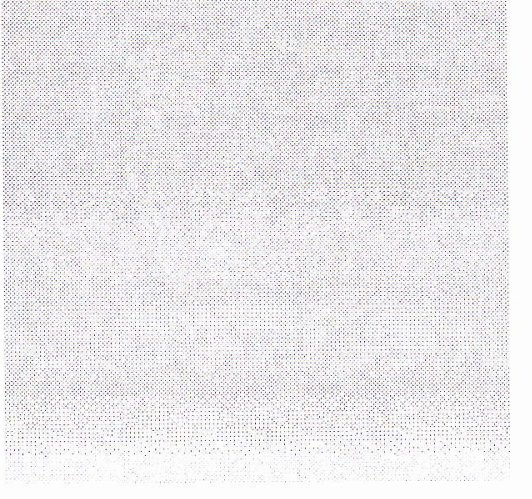

\author{
Por: Jorge E. Sierra*
}

\section{Resumen}

El objetivo de este trabajo es presentar y analizar los argumentos que Davidson desarrolla en contra de la noción representacionalista de conocimiento que, según él, hace posible que surja la disputa entre relativistas y realistas. El propósito de tales argumentaciones es hacer una defensa de una nueva forma de realismo mostrando que la pregunta por la existencia de un único esquema es en realidad una pseudopregunta, y que el relativismo no es, como muchos piensan, algo lógicamente posible.. La primera parte de este trabajo se ocupará de estudiar la crítica de Davidson a la noción representacionalista tradicional de verdad y conocimiento que da lugar al problema de la existencia del mundo externo, propio de la epistemología moderna. Si la concepción representacionalista de verdad implica que el problema de la existencia del mundo externo sea un genuino problema filósofico entonces, bastará con mostrar que tal problema es en realidad un seudoproblema para establecer que el representacionalismo es una postura equivocada. Para este fin abordaremos, en la segunda parte, un importante trabajo de David Stove en el que se examina la validez del problema del mundo externo en relación con el tema de la concepción representacionalista del conocimiento y su contraparte, el solipsismo que se fundamenta en el mito de lo subjetivo.

\section{Palabras Claves}

Racionalidad, esquemas conceptuales, Rorty, multiculturalismo, relativismo, verdad, neopragmatismo, antifundacionalismo, Davidson.

\section{Abstract}

In this article the author presents and analyzes Davidson's arguments against the notion of representation of knowledge which induced the dispute between relativists and realists. The contention supports a new form of realism for which the existence of a sole world scheme becomes a pseudo quest and that relativism despite its widespread acceptance isn 't possible in logical terms.

The first part of this work deals with Davidson's critic to the traditional notion of representation of truth and knowledge a topic that issued the problem of the existence of an outer world, a central issue in modern epistemology. If such conception implies that the existence of an outer world is a genuine philosophical problem, by establishing that it is just a pseudo-problem would prove representationism wrong.

In the second part, and based on David Stove's work, the topic of the outer world is examined as well as solipsism, its counterpart grounded on the myth of subjectivity.

\section{Keywords:}

Rationality, Conceptual schemes, Richard Rorty, Multiculturalism, Relativism, Truth, Neopragmatism, Antifoundationalism, Davidson.

Artículo: Recibido, 28 de julio de 2006; aprobado, 28 de Noviembre de 2006 


\section{INTRODUCCIÓN}

Muchos filósofos contemporáneos se han quejado tanto del creciente clima de irracionalidad presente en nuestra cultura como de su defensa filosófica por parte de ciertos intelectuales.' El motivo de la queja, independientemente de qué tan justificado sea, proviene del relativismo presente en ciertas tesis filosóficas que con tanto ahínco defienden intelectuales de tan diversas escuelas y posturas tales como Quine, Foucault, Goodman, Davidson, Rorty, Feyerabend y Kuhn. Para tales filósofos, en mayor o menor grado, la objetividad y la noción de verdad por correspondencia, propias del realismo filosófico, deben ser abandonadas. Pero para los realistas, dicho abandondono supone, ipso facto, entrar en las tenebrosas tierras del relativismo y la irracionalidad y abandonar, de este modo, la creencia fundamental en la existencia de criterios y métodos de justificación universales para nuestras creencias que no sean ni locales ni relativos a nuestra particular cultura.

Pero Żexiste un rasgo en común que posean todas nuestras creencias racionales? La discusión en torno al multiculturalismo parece abocarnos a una respuesta negativa y con ello, conducirnos al relativismo. Si cada cultura posee criterios internos para validar sus creencias entonces, es imposible que existan procedimientos universales de racionalidad. $Y$ en ausencia de tales procedimientos, hablar de la validez objetiva de nuestras creencias no pasa de ser eso, una simple forma de hablar. El reto que plantea el multiculturalismo se ve además acentuado por el el naturalismo biológico, ya que parece implicar una especie de bancarrota de la idea de racionalidad, pues nuestros esquemas conceptuales que ordenan lo dado en la experiencia y hacen posible el conocimiento, son contingentes desde un punto de vista evolutivo $y$, en consecuencia, no nos permiten suponer la existencia de un orden objetivo y racional del mundo. El multiculturalismo halla otro motivo a favor de su causa en los recientes debates en filosofía de la ciencia: la idea misma de racionalidad científica ha hecho crisis a partir de los trabajos de Kuhn y Feyerabend, quienes mostraron que no era posible tener una teoría pura de la racionalidad, pues, no había nada más allá de las múltiples prácticas culturales o históricas dentro de las cuales se había desarrollado las diversas teorías científicas, algo que pudiese denominarse como racionalidad científica a secas. Si sólo existían cánones locales de conocimiento, históricamente determinados y no universalizables, entonces el relativismo era una opción correcta y no habría manera de demostrar la validez de un discurso particular en contraposición a otro. Todos los discursos serían igualmente válidos y la tradicional separación entre discursos objetivos y racionales y discursos subjetivos e irracionales era imposible de trazar.

Cuando Thomas S. Kuhn publicó en 1962 su célebre obra La estructura de las revoluciones científicas, no previó la revolución que en el ámbito de la filosofía y de las ciencias humanas iba a producir al establecer la importancia de incluir el contexto de descubrimiento dentro del estudio filosófico de la ciencia. Si la historia de la ciencia era relevante para tener una visión más completa del trabajo de investigación de los científicos, ello permitiría ver que la ciencia no progresaba mediante acumulación, sino a través de revoluciones científicas.

Kuhn estableció que dado un conocimiento histórico más detallado de la ciencia, quizá fuese posible establecer que la ciencia no se desarrollaba, después de todo, mediante simple acumulación de descubrimientos. Y sugirió entonces, introducir el concepto de paradigma para una mejor comprensión del progreso científico. Por paradigma Kuhn entendía un grupo de creencias, hábitos y prácticas que determinaban la práctica de la ciencia durante un determinado período histórico.

Kuhn entonces explicó que el desarrollo de la ciencia se daba mediante revoluciones científicas, en las cuales se producía un cambio de paradigma por otro. Antes de la revolución se

1. Me refiero a autores como Thomas Nagel, Searle, Putnam y Habermas, quienes han desplegado influyentes argumentos en defensa de la racionalidad y en contra del relativismo. Las obras citadas de dichos filósofos en la bibliografía contienen numerosos pasajes en las que se expresan sus quejas en contra del irracionalismo actual. 
daba un período de ciencia normal en la que los científicos trabajaban bajo un paradigma común. Durante dicho período los científicos no buscaban nuevos hechos ni nuevas teorías, pues sólo se dedicaban a la resolución de ciertos rompecabezas. Cuando el paradigma entraba en crisis, debido a una serie de anomalías que no eran explicables dentro del paradigma, se producía un período de ciencia extraordinaria o revolución científica. Tal crisis se solucionaba mediante la elaboración de un nuevo paradigma pues, durante tal período de crisis se producía un aumento de nuevas teorías, una de las cuales era seleccionada como el nuevo paradigma. El logro más importante y problemático de Kuhn fue su tesis de la inconmensurabilidad entre paradigmas, la cual implicaba que era imposible traducir en un lenguaje común dos teorías inconmensurables. No existía, por tanto una, experiencia neutral para decidir la verdad entre dos teorías rivales. En este sentido, los científicos que vivían bajo paradigmas distintos, habitaban mundos distintos. Para muchos, este resultado conduce a una forma extrema de relativismo.

Si el multiculturalismo introduce la idea de que diferentes culturas interpretan el mundo cada una desde su peculiar esquema conceptual, entonces es importante preguntarse si tiene sentido la idea de hablar de un único esquema conceptual que garantice las pretensiones de validez objetiva de nuestras creencias o si por el contrario, ante la presencia de múltiples esquemas que interpretan la realidad a su manera, no es posible hablar de verdad objetiva. En un influyente artículo ${ }^{2}$, Davidson sugiere una posible salida al dilema de si existen múltiples esquemas o sólo un único esquema conceptual.

El relativismo, según Davidson, sólo tiene sentido dentro de un enfoque representacionalista del conocimiento y del lenguaje. De esta forma, el relativismo sólo es posible dentro de la idea del tercer dogma del empirismo, esto es, bajo el supuesto de que existe, por una parte, una realidad no interpretada y, por otra, un esquema conceptual determinado a partir del cual es interpretada dicha realidad desde determinada cultura. Si cada esquema conceptual interpreta, desde su particular visión, un conjunto de hechos dados, entonces es posible pensar que se den varias visiones alternativas de la forma de ser del mundo. Lo que Davidson propone es una manera de entender las nociones tradicionales de conocimiento, creencia y justificación sin necesidad de recurrir a la idea de representación. Cuando se hace esto, afirma Davidson, es posible entender por qué carece de sentido discutir acerca de qué cultura o esquema conceptual es el correcto, es decir, cuál es el que de verdad encaja con la realidad, pues no tiene sentido decir que hay muchos esquemas o decir que sólo hay uno.

El objetivo de este trabajo es presentar y analizar los argumentos que Davidson desarrolla en contra, no tanto de la cuestión misma de si hay un único esquema conceptual válido, como de la noción representacionalista de conocimiento que, según él, hace posible que surja la disputa entre relativistas y realistas. El propósito de ambas argumentaciones es hacer una defensa de una nueva forma de realismo mostrando que la pregunta por la existencia de un único esquema es en realidad una pseudopregunta, y que el relativismo no es, como muchos piensan, algo lógicamente posible, pues "si por relativismo conceptual queremos dar a entender la idea de que los esquemas conceptuales y los sistemas morales, o las lenguas asociadas con ellos, pueden diferir enormemente, hasta el punto de ser mutuamente ininteligibles o inconmensurables, o por siempre fuera del alcance de resolución racional, entonces rechazo el relativismo conceptual. ${ }^{13}$

La primera parte de este trabajo se ocupará de estudiar la crítica de Davidson a la noción representacionalista tradicional de verdad y conocimiento que da lugar al problema de la existencia del mundo externo, propio de la epistemología moderna. Si la concepción representacionalista de verdad implica que el problema de la existencia del mundo externo sea un genuino problema filósofico entonces, bastará

2. Davidson, D., "Sobre la idea misma de un esquema conceptual" en: D., De la verdad y de la interpretación, Barcelona, Gedisa, 1990.

3. Davidson, D., "El mito de lo subjetivo" en: Subjetivo, intersubjetivo, objetivo, Madrid, Cátedra, 2003, p. 73. 
con mostrar que tal problema es en realidad un seudoproblema para establecer que el representacionalismo es una postura equivocada. Para este fin abordaremos, en la segunda parte, un importante trabajo de David Stove en el que se examina la validez del problema del mundo externo en relación con el tema de la concepción representacionalista del conocimiento y su contraparte, el solipsismo que se fundamenta en el mito de lo subjetivo.

\section{DAVIDSON Y EL MITO DE LOSUBJETIVO}

Según Davidson, la concepción de la mente y su lugar en la naturaleza que, está detrás del problema acerca de la existencia del mundo externo, es aquella según la cual nuestro conocimiento se compone de dos elementos: los datos inmediatos de los sentidos, que son dados a la mente, y la actividad de interpretación o construcción que es propia del pensamiento. Ahora bien, al tratar de explicar la objetividad del conocimiento a partir de tal concepción de la mente surge inevitablemente el problema del escepticismo acerca de nuestro conocimiento del mundo externo. Pues, si no podemos describir los datos de los sentidos en un lenguaje libre de interpretación o carga teórica, entonces no podemos decidir qué creencias son objetivas porque corresponden con la forma de ser del mundo. La única forma de superar dicho problema sería apelar a "una fuente última de evidencia, el carácter de la cual pueda especificarse totalmente sin hacer referencia a aquello a favor de lo cual es evidencia." ${ }^{4}$

Pero la apelación a tal fuente supone que podemos describir los datos sensoriales $\sin$ necesidad de recurrir a lo que sucede a nuestro alrededor. Pero si esto es así, entonces la evidencia con la que contamos para nuestro conocimiento del mundo externo es problemática pues es posible, como diría Descartes, no sólo que nuestros sentidos nos engañen, sino que estemos sistematicamente equivocados acerca de todas nuestras creencias acerca del mundo. En resumen, según Davidson, llegamos a tal punto de vista problemático porque:

"...se piensa que es necesario aislar del mundo
externo las fuentes últimas de la evidencia a fin de
garantizar la autoridad de la evidencia para el
sujeto. Ya que no podemos estar seguros sobre
cómo es el mundo externo a la mente, lo subjetivo
puede mantener su virtud, su castidad, su certeza
para nosotros, solamente siendo protegido de la
contaminación del mundo. Un problema familiares
que la desconexión crea una brecha que ningún
razonamiento o construcción puede salvar de
manera plausible. Cuando se ha escogido este
punto de partida, no hay manera de decir de qué
son evidencia las evidencias, o eso es lo que
parece. Entonces nos amenazan el idealismo,
formas reduccionistas de empirismo y el
escepticismo."

Se supone entonces que las evidencias sobre las que descansan nuestras teorías o esquemas conceptuales son subjetivas de un modo insuperable. Pero si esto es así, todo lo que se base en ellas será igualmente subjetivo, es decir, nuestras creencias, nuestros deseos e incluso lo que queremos decir con nuestras palabras. Llegamos entonces a la paradójica situación según la cual "nuestras creencias pretenden representar algo objetivo, pero el carácter de su subjetividad no nos deja dar el primer paso para determinar si corresponden a lo que pretenden representar. ${ }^{16}$

Lo que Davidson nos ha mostrado hasta aquí, con esta línea de argumentación, es que el dualismo esquema-contenido es en realidad una de las formas del dualismo entre lo subjetivo y lo objetivo. Pero el hallazgo verdaderamente sorprendente es que ambos dualismos tienen un origen en común, a saber, una concepción de la mente con sus correspondientes estados y objetos privados. Si subjetividad significa la privacidad de lo mental, entonces, sugiere Davidson, es posible poner en entredicho la legitimidad de tales dualismos a partir de una versión revisada de la relación entre mente y mundo.

Tal revisión parte de nuestra comprensión acerca de lo que sabemos cuando conocemos el significado de una palabra $u$ oración. Tal

4. Ibid., p. 77.

5. Ibid.

6. Ibid., p. 78. 
comprensión indica que los significados "no están en la cabeza", es decir, que no son entidades privadas o subjetivas y que, en consecuencia,

"...en los casos más simples y más básicos, el significado de las palabras y de las oraciones se deriva de los objetos y las circunstancias en presencia de las cuales aquéllas se aprendieron. Si mediante el proceso de aprendizaje se ha condicionado a alguien a considerar verdadera una oración en presencia de fuego, la misma será verdadera (usualmente) cuando haya un fuego presente (...) Por supuesto que muchas palabras y oraciones no se aprenden de esta manera; pero son las que así se aprenden las que anclan el lenguaje al mundo."

Dado que los significados de los enunciados son proposiciones, y a su vez dichas proposiciones constituyen los objetos sobre las que versan las creencias, los deseos y las intenciones de los hablantes entonces, si los significados no son objetos privados, tampoco lo son los pensamientos expresados por medio del lenguaje. Dado este nuevo enfoque del pensamiento y del lenguaje, no es posible planetarse el tipo de dudas cartesianas que hacen posible el escepticismo acerca del mundo externo. Al abandonar el enfoque representacionalista del conocmiento, el significado y la creencia, el problema de si estamos representando con precisión el mundo que nos rodea deja de ser una cuestión filosóficamente interesante.

\footnotetext{
"Si las palabras y los pensamientos tratan necesariamente, en los casos más básicos, acerca de la clase de objetos y acaecimientos que comúnmente los causan, no hay lugar para las dudas cartesianas sobre la existencia independiente de tales objetos y acaecimientos.Puede haber dudas, por supuesto. Pero no necesita haber nada de lo que estemos indudablemente en lo cierto para que sea seguro que la mayoría de las veces estamos en lo cierto sobre la naturaleza del mundo." ${ }^{8}$
}

Pero ¿̇por qué no es posible que nuestras creencias estén sistemáticamente equivocadas acerca del mundo? La respuesta de Davidson es novedosa e implica una redefinición del concepto tradicional de creencia. Las creencias no son verdaderas o falsas porque representen $\circ$ no objetos en el mundo. Más bien se trata de entender que ciertas creencias básicas son causadas por los objetos sobre las que dichas creencias tratan, pues si no sucediese así el mismo conceto de creencia sería imposible. De esta forma, la posibilidad de que estemos equivocados acerca de todo

"...se descarta si aceptamos que los significados de nuestras oraciones más simples se dan mediante las situaciones que por regla general nos llevan causalmente a considerarlas verdaderas o falsas, ya que considerar que una oración que entendemos es verdadera o falsa es tener una creencia." ${ }^{\prime 9}$

De hecho, ni los datos de los sentidos ni tampoco los sentidos mismos juegan un papel determinante en la explicación de la creencia y el conocimiento. Se trata más bien de entender cómo los contenidos de la mente dependen causalmente de las complejas relaciones causales que se dan entre las actitudes proposicionales y el mundo externo.

Si lo dicho hasta aquí, es cierto, afirma Davidson, la epistemología no necesita ocuparse más de objetos que se dan de manera privada en el espacio interior de la mente. Si el esquema y el contenido se dan de manera conjunta en el proceso de conocimiento según el modelo representacionalista, entonces se pueden eliminar también conjuntamente. En conclusión,

\footnotetext{
"Una vez que damos este paso, no hay objetos con respecto a los cuales surja el problema de la representación. Las creencias son verdaderas o falsas, pero no representan nada. Es bueno librarse de las representaciones y con ellas de la teoría de la verdad por correspondencia, puesto que es pensar que hay representaciones lo que engendra indicios de relativismo."10
}

Una vez eliminado el tercer dogma del empirismo cae la polémica misma acerca de si estamos correctamente representando el mundo - no. Pero para explorar más a fondo esta cuestión es preciso adentrarnos en el tema del solipsismo, que como ya vimos resulta crucial

7. Ibid., p. 80 .
8. Ibid., p. 80 .
9. Ibid., p. 81 .
10. Ibid., p. 82 . 
comprender a fondo si se quiere entender la crítica al representacionalismo.

\section{EL EFECTO ISMAEL Y LA VALIDEZ DE LA PREGUNTA "¿EXISTE UN MUNDO EXTERNO?”}

Antes de explicar en qué consiste el efecto Ismael, es preciso dar un contexto mínimo de discusión acerca del problema del mundo externo.

Pero ટ̇existe un problema acerca de la realidad - existencia del mundo externo? Por lo menos desde Descartes se pensó que la cuestión escéptica acerca de la existencia del mundo externo era un problema real y genuino que la filosofía debía responder bajo la forma de una demostración o, dado el caso de que esto no se lograra, que deberíamos contentarnos con aceptar su existencia como un mero artículo de fe o como una creencia que era útil para nuestros asuntos de la vida práctica. Para otros, en cambio, la pregunta acerca de la existencia de un mundo externo no plantea en realidad un genuino problema que deba o pueda responderse. Desde esta óptica el problema es si hay un problema o una cuestión a la qué responder. Descartes, Locke, Berkeley, y Kant, entre otros, consideraron que sí había un problema que solucionar, mientras que para Davidson será más bien un pseudoproblema cuya "solución" será su disolución.

Como bien lo anota Gabriel, se pueden distinguir dos maneras de tratar el problema:

\footnotetext{
"Antes que nada, conviene hacer una distinción importante: por "ser" no se entenderá aquí el «ser-así» (el modo de ser), sino el "ser-ahí (la existencia). Lo distinción es crucial, porque permite atribuir al mundo exterior una existencia, un ser-ahí independiente, sin con ello afirmar que dicho mundo exterior se halla formado en su ser-así, tal como a nosostros se nos manifiesta."
}

De esta forma, se puede preguntar si existe un mundo externo o se puede preguntar si el mundo externo es tal como lo conocemos, esto es, si nuestro conocimiento de él es objetivo o no lo es. En el primer caso la cuestión es si sabemos o no que hay un mundo externo independiente del sujeto, mientras que en el segundo, la cuestión de la existencia se desplaza a la cuestión de saber cómo es ese mundo externo. Una cosa es preguntar si sabemos o podemos estar seguros de que hay un mundo externo cuya realidad sea independiente del sujeto, y otra cosa es preguntar si sabemos que el mundo externo es tal como nos lo representamos, donde se asume que hay un mundo externo.

Ahora bien, la disputa entre el realismo y el idealismo puede ser entendida como un asunto tocante a la esencia o constitución del mundo externo y su existencia, esto es, si el mundo externo es material o inmaterial, o si existe o no existe. Para un realista decir que el mundo es inmaterial implica negar la existencia del mundo externo, en tanto que para un idealista asumir que la independencia o realidad del mundo externo depende de su constitución material implica negar la existencia del mundo externo (recuérdese la polémica Locke-Berkeley). Independientemente de quien tenga la razón, lo claro es que ambas posiciones no niegan la existencia o realidad del mundo externo; ambos bandos explican la realidad del mundo externo apelando a su constitución y ese es el punto en disputa. Pero también es posible entender que el realismo afirme que existe el mundo externo sin necesidad de que su afirmación esté encuadrada dentro del marco de una doctrina-velo tal como supone el representacionalismo, y como sucede, en general, con el realismo y el idealismo modernos.

De la misma manera que se puede dudar de la existencia del mundo externo, se puede negar su existencia. Como hemos visto ni el realismo ni el idealismo niegan que exista un mundo externo, aunque ambas posiciones se acusen entre sí de negarlo o por lo menos de hacer dudosa su existencia. Si esto es así, existe una tercera posición que pueda ser contraria tanto al realismo como al idealismo, a saber, el solipsismo en tanto que su posición consiste en negar que existe un mundo externo. Obviamente los idealistas han

11. Gabriel, Gottfried, "El «escándalo de la filosofía». El problema de la realidad y su disolución" en: Isegoría, 7 , 1993, pp. 107-121,p. 107 y 108. 
sido acusados de solipsismo porque se dice que su doctrina implica convertir la realidad del mundo externo en mera ilusión. Pero tal acusación es injusta si se tiene en cuenta que el sujeto del idealismo no es el sujeto del solipsismo. El idealista afirma "Existe un mundo externo y su constitución es inmaterial", mientras que el solipsista afirma "Sólo existo yo" o "No existe el mundo externo, sólo existo yo". Es claro que ambas posiciones no afirman lo mismo

El nombre de efecto Ismael es tomado por Stove a partir de una situación hipotética, construida con base en el relato de Moby Dick, en el que un tripulante (Ismael) de un barco logra escapar con vida y afirma: "Sólo yo he escapado para contártelo". La situación hipotética que se nos pide que pensemos es la siguiente:

"Pero imaginemos que un hombre nos dijera que había estado a bordo de un barco y que, en un encuentro con una ballena, habían muerto todos los tripulantes del barco. Su afirmación tendría un grave defecto, de una clase peculiar a la que yo llamo (permitiéndome una ligera licencia) el efecto Ismael: y es que si su afirmación fuera cierta, no podría haberla hecho. Sería más o menos como si un hombre nos dijera con rugido: "Sólo puedo hablar susurrando" o si alguien nos dijera "No sé decir ni una sola palabran."

Por lo que respecta a las afirmaciones, el efecto Ismael consiste en hacer una afirmación, que dado el caso de que sea verdadera, ni debería hacerse ni podría hacerse. Así, en el caso de la anterior situación hipotética, si Ismael afirma "Todos los tripulantes del barco murieron" está diciendo algo que no puede decirse si su afirmación es cierta, o lo que es igual, Ismael sólo puede hacer esa afirmación si y sólo si alguien del barco ha sobrevivido. Pero también es posible encontrar preguntas que estén afectadas por el efecto Ismael. Si Ismael pregunta "¿̇Sobrevivió alguien del barco?", él no puede hacer esa pregunta si no a condición de que la respuesta sea sí, es decir, sólo si alguien del barco sobrevivió. Stove aclara que las afirmaciones o preguntas con efecto Ismael no dependen del hecho de que su expresión sea pública. Ismael no puede preguntarse $\circ$ decirse a sí mismo o a otros si sobrevivió alguien del barco a menos de que alguien del barco haya sobrevivido. Stove establece dos hechos acerca de las preguntas Ismael. Primero, que existe una condición previa para la formulación de tales preguntas, esto es, que para poder realizar la pregunta la respuesta a ella debe ser sí. Segundo, que quien la formula debe saber que la respuesta es sí. Y si esto es así, quiere decir que la pregunta no puede ser sincera, pues cuando se hace una pregunta genuina $y$ sincera se ignora su respuesta.

Aclarado en qué consiste el efecto Ismael, veamos lo que busca demostrar Stove.

"Este ensayo es una defensa de la necesidad del realismo. Mejor dicho: es una defensa de la inevitabilidad del realismo; aunque, como se verá, estoy muy lejos de creer que tal necesidad sea sólo pragmática. Espero demostrar, primero, que la negación, inlcuso la duda, de la existencia de un mundo externo es una víctima impotente del efecto Ismael (...) Segundo, trataré de demostrar que "Existe un mundo externo" no es una teoría científica ni una hipótesis inductiva, y que, casi con toda seguridad, ni siquiera es una afirmación contigente." ${ }^{13}$

Por ahora sólo vamos a considerar el primero de los puntos que Stove quiere tratar; el segundo será tratado más adelante.

Veamos cómo demuestra Stove que la pregunta por la existencia del mundo externo es una pregunta Ismael. El primer paso consiste en mostrar que la pregunta " $\dot{E}$ Existe por lo menos un ser humano?" es una pregunta Ismael. Si un ser humano se hace esa pregunta, él no podría formularla a menos que la respuesta no sea sí. En este caso se trata de una pregunta Ismael sólo si la formula un ser humano. Pero es claro que esta "pregunta" no puede ser una pregunta genuina, sino más bien insincera. $Y$ como ya vimos no puede formularse a menos de que la respuesta no sea sí. Pero aquí Stove hace una observación importante. Si bien la proposición "Existe por lo menos un ser humano" no es una proposición necesaria les claro que es una proposición contingente, pues no es una verdad necesaria que existan seres humanos), sí tiene que ser necesario que exista por lo menos un ser humano como

12. Stove, Barry, El culto a Platón y otras locuras filosóficas, Madrid, Cátedra, 1993, p. 93.

13. Ibid., p. 98. 
condición para que alguien pueda preguntarse si es cierto que existe por lo menos un ser humano. La pregunta "¿̇Existe por lo menos un ser humano?" sólo puede hacerse si y sólo si hay un ser humano, esto es, es una condición necesaria que si alguien pregunta por la existencia de un ser humano, exista por lo menos un ser humano. ${ }^{14}$

Ahora veamos qué tiene que ver la pregunta Ismael de si existe por lo menos un ser humano con la cuestión de la existencia del mundo externo. Para Stove "el realismo es...sencillamente la afirmación de que existe un mundo externo". ${ }^{15} \mathrm{De}$ lo que se trata es de establecer qué es necesario y qué es suficiente para que la afirmación "Existe un mundo externo" sea verdadera (o para que el realismo sea verdadero). No es lógicamente necesario para que el realismo sea verdadero que exista una mesa o un tomate o que existan mesas y tomates. Esto es lo mismo que afirmar que es posible que exista un mundo externo que no contenga tales objetos. Esto queda mejor expresado en las siguientes implicaciones. La verdad de "Existe un mundo externo" no esta implicada en la proposición conjuntiva "Existe una mesa, y un tomate, y...", ni en la proposición disyuntiva "Existe una mesa, o un tomate o..." En el caso de la proposición conjuntiva, bastaría con que no existiera un tomate para que tal proposición fuera falsa, con lo cual sería falsa la proposición "Existe un mundo externo". Pero es claro que la falsedad de "Existe un mundo externo" no se sigue de la falsedad de la conjunción mencionada. En el caso de la disyunción la cosa es un poco más compleja. Pues no se sigue de forma necesaria que exista un mundo externo de la proposición "existe o bien un tomate o bien una mesa" porque puede ser el caso de que no haya ni mesas ni tomates (es decir, que la disyunción sea falsa) y no obstante exista un mundo externo. Pero si bien la conjunción y la diyunción mencionadas no son una condición necesaria para que exista un mundo externo, sí son una condición suficiente para su existencia. Esto quiere decir que si la proposición "Hay un tomate en esta estantería y una mesa en la otra habitación" es verdadera, entonces es verdadera la proposición "Existe un mundo externo". La misma conclusión se sigue si existe un tomate o una mesa cualquiera, esto es, la verdad de "Existe por lo menos un tomate o una mesa" implica la verdad de "Existe un mundo externo". ${ }^{16}$

Ahora bien, si "Existe por lo menos un tomate" implica "Existe un mundo externo", esto es si cualquier proposición existencial singular verdadera demuestra la verdad de la existencia de un mundo externo, eso quiere decir que de la proposición "Existe por lo menos un ser humano" se sigue "Existe un mundo externo". Veamos el argumento que con base en lo anterior demuestra que la pregunta por la existencia de un mundo externo es una pregunta Ismael.

\footnotetext{
"Pero, necesariamente, ningún ser humano podría preguntar, ni siquiera interiormente, si existe un mundo externo, si no existe un ser humano por lo menos. $Y$ necesariamente, si existe por lo menos un ser humano, existe un mundo externo. Por tanto, necesariamente, ningún ser humano podría preguntar si existe un mundo externo a menos que exista un mundo externo. La pregunta de si existe un mundo externo es, en consecuencia, una pregunta Ismael, y de las peores. Es lógicamente imposible que un ser humano formule esta pregunta, siquiera interiormente, y que la respuesta no sea «sí». No sólo es preciso que la respuesta sea «sí»; cualquiera que no esté bajo la influencia de alguna enfermedad mental terrible tiene que saber que la respuesta es «sí». Si alguien que no tenga la mente enferma pregunta si existe un mundo externo, su pregunta sólo puede ser insincera, en el sentido de que en realidad no busca la información que parece
}

14. Ibid., p. 102 y 103.

15. Ibid., p. 104. Recuérdese lo que dijimos en la introducción: es posible formular el realismo ("Existe un mundo externo") sin necesidad de llegarse a plantear la naturaleza o constitución del mundo externo. De Hecho, Stove rechaza todas aquellas doctrinas-velo por considerar que también son víctimas del efecto Ismael, esto es, son doctrinas que pretenden decir lo que no puede decir coherentemente. El siguiente pasaje de Stove es una ilustración de por qué las doctrinas-velo implican afirmaciones Ismael: "Quiero decir doctrinas tales que hacen que un velo impenetrable nos separe del conocimiento del universo real; o tales que nos colocan un obstáculo insuperable que nos impide trepar al único lugar desde el cual podríamos ver al cosmos tal cual es. Puesto que cuentan lo que hay del otro lado, o por lo menos cuentan que hay otro lado en un velo que, también lo dicen, ninguna mente humana puede atravesar, todas las doctrinas velo están mortalmente afectadas por el efecto Ismael." (Ibid., p. 95)

16. Ibid., p. 104 y 105.

17. Ibid., p. 105 y 106. 
buscar."17

Analicemos ahora la estructura de este argumento.

1. Necesariamente ningún ser humano puede preguntar si existe un mundo externo a menos que exista un ser humano.

2. Por lo tanto, existe por lo menos un ser humano. (por la premisa 1)

3. Necesariamente si existe por lo menos un ser humano, entonces existe un mundo externo.

4. Por lo tanto, existe un mundo externo. (A partir de 2 y 3 por modus ponens).

5. Por lo tanto, necesariamente ningún ser humano podría preguntar si existe un mundo externo a menos que exista un mundo externo. [A partir 1, 2, 3, 4 por las propiedad transitiva de la implicación, es decir, (1) si necesariamente ningún ser humano puede preguntar si existe un mundo externo a menos que exista un ser humano (si $p$, entonces q), lo que implica que (2) existe por lo menos un ser humano. $Y(3)$ si existe un ser humano implica que (4) existe un mundo externo/y si q entonces r), entonces (5) necesariamente ningún ser humano podría preguntar si existe un mundo externo a menos que exista un mundo externo (entonces si p, entonces $r$ )].

La conclusión de este argumento demuestra que la pregunta por la existencia de un mundo externo es una pregunta Ismael, pues la posibilidad misma de formularla implica necesariamente que sea verdadera la proposición que quiere ponerse en duda, lo que es equivalente a decir que es lógicamente imposible que algún ser humano la formule y la respuesta no sea sí, pues si así no fuera estaríamos afirmando que el mundo externo existe y que no existe. Es necesariamente falso que alguien formule la pregunta por la existencia del mundo externo y se niegue a reconocer la condición que hace posible formular la pregunta, lo cual es absurdo. Por lo tanto, si la pregunta "¿̇Existe un mundo externo?" es una pregunta Ismael, entonces eso quiere decir que quien la formula debe saber que la respuesta es verdadera, y si sabe que su pregunta intenta preguntar lo que hace posible hacer la pregunta, sus intenciones no pueden ser sinceras. De esta forma, la pregunta "¿̇xiste un mundo externo? es en realidad una pseudopregunta que cuando se expresa implica una especie de autorrefutación del escéptico nacida de su falta de sinceridad al formularla. Esto indica también que la cuestión de la existencia del muno externo no puede ser un problema genuino: al demostrar que la pregunta de si existe un mundo externo presupone lo que quiere ponerse en duda $\circ$ negarse, no tiene sentido buscar una solución para algo que se sabe que es verdadero. Al ser una pregunta Ismael, se diluye la pseudocuestión de la existencia de un mundo externo.

No obstante, el argumento de Stove deja algunos puntos sin aclarar. Por ejemplo, no es evidente que "Existe por lo menos un ser humano" implique la verdad de "Existe un mundo externo", aunque sea claro que quien afirme o pregunte si existe un ser humano necesariamente debe comprometerse con la existencia de por lo menos un ser humano. Puede ser posible que existan seres humanos y no exista un mundo externo. Obviamente si se pregunta "¿̇Cómo sabemos que existen seres humanos" implica afirmar que existe por lo menos un ser humano. Pero si preguntamos "¿̇Existe por lo menos un tomate o una mesa?", tal pregunta no es una pregunta Ismael, de ahí que no se siga que deba existir un tomate o una mesa para que la pregunta pueda realizarse. Pero como hemos visto, para Stove la verdad de "Hay por lo menos un repollo o un rey" implica "Existe un mundo externo". La pregunta que un escéptico podría plantear es cómo podemos estar seguros de que "Hay por lo menos un repollo o un rey".

\section{LA IMPOSIBILIDAD LÓGICA DEL SOLIPSISMO Y LANECESIDAD DELREALISMO.}

¿̇Expresa el realismo un proposición necesaria cuando afirma que existe un mundo externo? Como anota Stove del hecho de que la pregunta de si existe un mundo externo es una pregunta Ismael no se sigue que la afirmación "Existe un 
mundo externo" sea lógicamente necesaria. Como vimos antes, no se sigue de la pregunta Ismael "¿̇Existe por lo menos un ser humano?" que sea necesario que existan seres humanos, sino mas bien que es necesario para que la pregunta pueda hacerse que exista por lo menos un ser humano. De la misma manera, la proposición "Existe un mundo externo" puede ser una proposición contingente así sea necesario que para que alguien pregunte por la existencia de un mundo externo sea cierto que exista un mundo externo. Pero según Stove existe otra razón para pensar que la proposición "Existe un mundo externo" no sea contigente, a saber, que si tal afirmación no tiene ningúna proposición contigente que sea su contraria, entonces por las definiciones mismas de las modalidades, tal afirmación sería necesaria. Una proposición es contigente si es posible negarla sin contradicción, esto es, si tiene alguna proposición contraria contigente. $Y$ una proposición es necesaria si no es posible que tenga alguna proposición contraria, esto es, si su negación implica una contradicción. De esta forma, las verdades necesarias no tienen alternativas posibles: si son ciertas, son necesariamente cientas; por el contrario, las verdades contingentes tienen alternativas posibles: si son ciertas, son posiblemente ciertas.

Pero veamos por qué para Stove la proposición "Existe un mundo externo" es necesaria al no tener posibles proposiciones (o alternativas) contrarias que sean contingentes. $\dot{2}$ Es el idealismo objetivo una alternativa posible al realismo? La tesis del realismo es "Existe un mundo externo y su constitución es mental o espiritual". Ahora bien, para que esta afirmación fuera contraria contingente a la tesis del realismo ("Existe un mundo externo") ella no tendría que afirmar la tesis del realismo como parte de lo que dice. La negación contingente de " $P$ " no es "P $y$. .", sino "no-P y...". Por lo tanto el idealismo objetivo no es un contrario contigente $o$ una alternativa posible al realismo porque ambas posiciones no expresan proposiciones contrarias. ${ }^{18}$
Como indicamos en la introducción, la única alternativa posible al realismo es el solipsismo. Este afirma "No existe un mundo externo", es decir niega la tesis del realismo, esto es, la proposición "Existe un mundo externo". Ahora bien, para demostrar que la tesis del realismo es una verdad necesaria se debe mostrar que el solipsismo es lógicamente imposible o necesariamente falso. Si $P$ es necesariamente falsa, no-P será necesariamente verdadera. De esta forma la proposición "Existe un mundo externo" no tendrá ninguna alternativa lógicamente posible y será una verdad necesaria. ${ }^{19}$

¿Qué es exactamente lo que se dice cuando se afirma que el solipsismo es lógicamente posible? Para algunos, decir que el solipsismo es lógicamente posible es lo mismo que decir que "Sólo existimos nosostros" no implica una contradicción. Es posible que el mundo externo sea algo irreal y que la única verdad sea que sólo existimos nosostros. Se supone que tal afirmación puede hacerse sin contradicción. Pero, según Stove, en esta afirmación se esconde lo que él llama el pronombre-problema. ${ }^{20}$ En la afirmación "Sólo existimos nosotros", el pronombre "nosostros" significa "cada uno de nosotros", en consecuencia lo que la afirmación dice es "Cada uno de nosostros es lo único que existe". Pero si se dice que cada uno de nosostros es lo único que existe, lo que dice implica afirmar un contrasentido. En consecuencia, este tipo de solipsismo no es lógicamente posible porque lo que afirma es necesariamente falso 0 autocontradictorio. De esta forma, el solipsismo que se intenta expresar al utilizar el pronombre "nosostros" es incoherente. De hecho, cualquier forma de solipsismo que utilice alguna forma de pronombre personal en plural o un equivalente suyo tiene que ser necesariamente falso.

¿Qúe se requiere entonces para expresar un verdadero solipsismo?

"Para que haya una versión contingente del solipsismo,

18. Ibid., p. 107

19. Ibid..

20. Ibid.

21. Ibid., p. 112. 
el pronombre que se debe usar debe estar en auténtica primera persona del singular. La afirmación debe ser "Sólo existo yo" o "No existe nada excepto yo»."

Ahora la cuestión es ver si el solipsismo correctamente formulado puede ser lógicamente posible. Se supone que el solipsismo pretende negar la existencia de algo en lo que pueden creer aquellos que no son solipsistas. Según Stove para que algo sea solipsismo debe expresar una ontología reducida, es decir, debe negar la existencia de alguna cosa que el no solipsista crea que de verdad existe. Ahora bien, la tesis de Stove es que el solipsismo no cumple con la condición de negar algo, es decir, no expresa una ontología reducida. Veamos cómo demuestra Stove que la afirmación del solipsismo "No existe nada excepto yo" no niega la existencia de nada. Se supone que la anterior frase niega en primer lugar niega la existencia de algo ("No existe nada...") y después afirma la existencia de algo ("...excepto yo"). Ahora bien, para que la frase "No existe nada excepto yo" niegue realmente algo debe decir o dar alguna información que permita identificar la excepción que se dice que existe. Si la frase no permite identificar claramente qué tanto es aquello que se niega que existe y qué tanto es aquello que se afirma que existe, no es posible que la frase niegue algo porque para poder saber qué es lo que niega debemos saber la magnitud de aquello que no niega. Si alguien dice "No existe nada excepto todo lo que existe" obviamente no estó negando la existencia de nada. Algo similar ocurre con la frase "No existe nada excepto yo", pues aquí la palabra "yo" no da ninguna información que permita saber a ciencia cierta cuál es la magnitud de lo que existe y así saber qué es lo que se niega que existe. $Y$ es que la palabra "yo" no tiene una referencia precisa que permita identificar la magnitud de aquello que abarca. En consecuencia, la frase no niega la existencia de nada porque no es posible saber exactamente a qué se refiere la palabra "yo". La información contenida en la frase es nula. De hecho, "yo" puede ser cualquier cosa que queramos: Dios, el cosmos, etc. En el caso de que la frase sea "No existe nada excepto David Stove" puede que exprese una ontología reducida, pero sólo a condición de que no sea todo lo que existe, pues en caso contrario no se está negando la existencia de nada: ¿́qué es exactamente lo que no existe fuera de David Stove? En conclusión:

"De forma que «No existe nada excepto yo»no niega la existencia de nada. De forma que no expresa una ontología reducida. De forma que no es solipsismo."22

Pero supóngase que es posible identificar a qué se refiere "yo" para que la frase "No existe nada excepto yo" exprese una ontología reducida y niegue algo. La identificación tiene que ser tal que excluya la posibilidad de que "yo" no pueda referirse sino a la excepción y que esta excepción no sea, por ejemplo, la totalidad de todo lo que existe. La frase se convierte entonces en "No existe nada excepto yo, y yo soy algo menos que todo lo que existe", que es claramemente una frase autocontradictoria. Así, cuando la frase expresa un verdadero solipsismo (una ontología reducida) inmediatamente produce un enunciado necesariamente falso o contradictorio.

Pero para Stove lo dicho hasta aquí es insuficiente para demostrar que "Existe un mundo externo" es una verdad necesaria. En primer lugar, no se ha demostrado que sea imposible una versión contigente del solipsismo. Se ha demostrado que el solipsismo no es una verdad necesaria, pero no sabemos si es posible que sea contingentemente verdadero otra versión suya. Sólo se han dado razones para pensar que es así. En segundo lugar, tampoco la "demostración" de que el solipsismo es el único contrario contingente del realismo es exhaustiva. Incluso, si la demostración de ambas cosas es concluyente queda por decidir si el realismo no es contingente. Así si el antecedente de la proposición "El solipsismo es lógicamente imposible, entonces el realismo es lógicamente necesario" es verdadero, su conclusión también lo es. Pero, aún suponiendo que el solipsismo sea lógicamente posible, es decir probablemente verdadero, y que, en consecuencia, el realismo sea contingente $\circ$ probablemente verdadero, es claro, según Stove, 
"...que la pregunta de si el realismo es cierto -si existe un mundo externo-- es una pregunta que ningún ser humano con la cabeza como Dios manda puede llegar a preguntar sinceramente."

\section{CONCLUSIÓN}

Lo que resulta interesante en el tratamiento que hace Stove del status del problema escéptico de si existe un mundo externo, es el modo en que convierte lo que es, supuestamente una ventaja para el escéptico, en una debilidad, a saber, el juego de las posibilidades lógicas que él pone en marcha para restarle credibilidad a ciertas afirmaciones. El escéptico nos dice que es lógicamente posible que estemos soñando, que es lógicamente posible que siempre estemos alucinando, que es lógicamente posible que el mundo externo no exista, o que es lógicamente posible que su existencia sea dudosa, que es lógicamente posible el solipsismo. Pero Stove nos muestra que es lógicamente imposible poner en duda o negar la existencia de un mundo eterno, que hay buenas razones para creer que es lógicamente imposible el solipsismo De alguna manera tambíen demuestra que es lógicamente imposible cualquier teoría del conocimiento que afirme ser una doctrina-velo, una doctrina representacionalista, pues, implica afirmar, a la vez, que podemos saber algo que ella misma dice que no podemos saber. Pero tal vez lo más valioso de la reflexión de Stove es que muestra que cualquier teoría del conocimiento que pretenda resolver el "problema" de la existencia de un mundo exterior y considere que la cuestión es genuina, tiene que ser, por fuerza, una teoría equivocada. Pero al afirmar esto, se está diciendo que el escepticismo acerca del mundo externo puede ser derrotado sin necesidad de apelar a una doctrina filosófica que pretenda servir de fundamento al conocimiento bajo la forma de un discurso totalizante, que nos exponga aquellas verdades universales, necesarias e incorregibles que hacen posible el conocimiento.

Tanto para Davidson como para Stove la función de la epistemología no es la de responder el reto del escéptico acerca del mundo exterior. La explicación del concepto de creencia presupone entender el contenido de las creencias básicas no como un objeto privado y subjetivo, sino como un evento $u$ objeto público. Las creencias no son representaciones que sirvan de mediadoras entre los sujetos y el mundo.

\section{BIBLIOGRAFÍA}

Arenas, L., et. al. (eds.), El Desafío del Relativismo, Madrid, Trotta, 1997.

Davidson, D., De la verdad y de la interpretación, Barcelona, Gedisa, 1990

$\overline{2003 .}$

Subjetivo, intersubjetivo, objetivo, Madrid, Cátedra,

Problems of Rationality, Oxford, Oxford University Press, 1998.

Devitt, M., Realism and Truth, New Jersey, Princeton University Press, 1991.

Eagleton, Terry, La idea de cultura, Barcelona, Paidos, 2001.

Gabriel, Gotffried, "El "escándalo de la filosofía». El problema de la realidad y su disolución" en: Isegoría, 7, 1993, pp. 107-121.

Goodman, N., Maneras de Hacer Mundos. Madrid, ed. Visor, 1990.

Laudan., L., Ciencia y relativismo, Madrid, Alianza, 1998.

Nagel, T., Una Visión de Ningún Lugar, México, F.C.E., 1996.

Papineau, D., Reality and Representation, Cambridge-Mass, Blackwell, 1987.

Putnam, H., Razón, Verdad e Historia, Madrid, ed. Tecnos, 1988. $\overline{1991 .}$

El Significado y las Ciencias Morales, México, U.N.A.M.,

Quine, W.V.O., "La relatividad ontológica", La Relatividad Ontológica y Otros Ensayos, Madrid, Tecnos, 1986, pp. 43 92.

Rorty, R., Consecuencias del Pragmatismo, Madrid, Tecnos, 1996.

La Filosofía y el Espejo de la Naturaleza, Madrid, Cátedra, 1989. $\overline{1996 .}$

Objetividad, Relativismo y Verdad, Barcelona, Paidós,

Verdad y progreso, Barcelona, Paidós, 2000. 